\title{
Further Asymptotic Expansions for the Error Functional
}

\author{
By B. W. Ninham* and J. N. Lyness**
}

Abstract. The generalised Euler-Maclaurin summation formula for the error functional $E f=R f-I f$ is not suitable for numerical computation if $f(x)$ contains singularities in the complex plane which lie close to the interval of integration. In this paper, we use the methods and results of an earlier paper to construct alternative asymptotic expansions suitable in such cases.

1. Introduction. In a previous paper, [1], we derived generalisations of the EulerMaclaurin summation formula. Asymptotic expansions for the error functional

$$
E f=R f-I f
$$

were constructed in cases for which the integrand $f(x)$ has singularities on the interval of integration.

It is well known that the effective use of conventional quadrature methods is difficult when the integrand has singularities close to the interval of integration in the complex plane. This is related to the fact that in this case the Euler-Maclaurin formula yields a rapidly diverging asymptotic expansion; (see [1]). Thus, for small $\epsilon$ the numerical integration of the simple function $1 /(x+\epsilon)^{1 / 2}$ over the interval $[0,1]$ by standard methods is difficult.

In this paper we derive alternate asymptotic expansions to the Euler-Maclaurin formula which are useful in such circumstances and which converge rapidly if $m$, the number of function evaluations, is small. The main features of our method for constructing asymptotic expansions are illustrated in Section 2 for a simple function and an end-point trapezoidal rule $R^{[m, 1]}$. In subsequent sections the integration rules are examined for functions which are integrable on $[0,1]$, which have singularities at $x=-\epsilon$ or $x=-\epsilon \exp [ \pm i \theta]$, and which may have additional algebraic singularities at $x=0$ and $x=1$. Thus we shall consider integrals of the form

$$
\text { If }=\int_{0}^{1} t^{\beta}(1-t)^{\omega}\left(t^{\eta}+\epsilon\right)^{\rho} h(t) d t ; \quad \eta>0,
$$

where $h(t)$ and its derivatives are continuous in $[0,1],|\arg \epsilon|<\pi$, and the exponents are restricted only to the extent that the integral exists. In our discussion below, the numbers $\beta, \omega$, and $\rho$ of (1.2) are assumed to be real; nevertheless, with obvious modifications, the results and derivations hold for complex exponents. The section headings indicate the rule and the function whose error functional is considered in that section.

Received July 19, 1967, revised March 13, 1968.

* One of the authors (B.W.N.) acknowledges the support of the Government of the Commonwealth of Australia by the award of a Queen Elizabeth II post doctoral fellowship.

** Work performed in part under the auspices of U.S. Atomic Energy Commission. 
We note that our treatment is sufficiently general to provide a new class of asymptotic expansions for the calculation of the hypergeometric function by means of its integral representation

$$
F(a, b ; c ; z)=\frac{\Gamma(c)}{\Gamma(b) \Gamma(c-b)} \int_{0}^{1} t^{b-1}(1-t)^{c-b-1}(1-t z)^{-a} d t
$$

(Erdélyi et al. [2]).

II. $R^{[m, 1]}:(x+\epsilon)^{\rho}$. In [1] we considered expansions of the error functional

$$
E f=R f-I f
$$

for a function $f(x)$ having singularities on the integration interval; here $R f$ denotes the result of an arbitrary quadrature rule. Consider the function

$$
q(x)=(x+\epsilon)^{\rho}, \quad \rho<0,
$$

where $\epsilon$ is small and positive. For the end-point trapezoidal rule $R^{[m, 1]}$ we know (see [1], Eq. (3.15))

$$
E^{[m, 1]} q=R^{[m, 1]} q-I q=\sum_{r=-\infty}^{\infty} \int_{0}^{1}(t+\epsilon)^{p} e^{2 \pi i r m t} d t .
$$

We write

$$
E^{[m, 1]} q=E_{0}{ }^{[m, 1]} q-E_{1}^{[m, 1]} q
$$

where

$$
E_{n}^{[m, 1]} q=\sum_{r=-\infty}^{\infty} \int_{n}^{\infty}(t+\epsilon)^{\rho} e^{2 \pi i r m t} d t ; \quad n=0,1 .
$$

Asymptotic expansions for both $E_{0}{ }^{[m, 1]} q$ and $E_{1}{ }^{[m, 1]} q$ follow most easily by successively integrating by parts. After summing over $r$, we have

$$
E_{0}^{[m, 1]} q \cong-2 \epsilon^{\rho+1}\left[\frac{\rho \zeta(2)}{(2 \pi m \epsilon)^{2}}-\frac{\rho(\rho-1)(\rho-2) \zeta(4)}{(2 \pi m \epsilon)^{4}}+\cdots\right]
$$

and

$$
E_{1}{ }^{[m, 1]} q \cong-2(1+\epsilon)^{\rho+1}\left[\frac{\rho \zeta(2)}{(2 \pi m(1+\epsilon))^{2}}-\frac{\rho(\rho-1)(\rho-2) \zeta(4)}{(2 \pi m(1+\epsilon))^{4}}+\cdots\right]
$$

The second of these expansions is suitable for numerical computation for moderate values of $m$. However, the first is unsuitable unless $m$ is large. Clearly, unless $m>|\rho / 2 \pi \epsilon|$, each term in (2.6) is larger than the previous term. Obviously these expansions are valid for all $\rho$.

Our general problem is adequately illustrated by this simple example. The existence of a branch point or pole of $f(x)$ close to the interval of integration leads to an Euler-Maclaurin expansion for the error functional which diverges badly, and is not suited for numerical computation. Stated in other terms, low degree polynomial approximation fails. We therefore derive an alternative expansion for $E_{0}^{[m, 1]} q$ which is useful for small $m \epsilon$.

To do this we obtain first an integral representation for $(t+\epsilon)^{\rho}$. Since [3] 


$$
\begin{aligned}
& \int_{0}^{\infty}(t+\epsilon)^{\rho} \epsilon^{p-1} d \epsilon=t^{p+\rho} \frac{(-\rho-p-1) !(p-1) !}{(-\rho-1) !} \\
& 0<\operatorname{Re}(p)<-\rho,|\arg \epsilon|<\pi,
\end{aligned}
$$

we have from the inversion formula for Mellin transforms that

$$
(t+\epsilon)^{\rho}=\frac{1}{2 \pi i} \int_{c-i \infty}^{c+i \infty} t^{\rho+p} \frac{(-\rho-p-1) !(p-1) !}{(-\rho-1) ! \epsilon^{p}} d p ; \quad 0<c<-\rho .
$$

Substituting (2.9) into (2.5), we find

$$
E_{0}^{[m, 1]} q=\frac{1}{\angle \pi i} \sum_{r=1}^{\infty} \int_{0}^{\infty} d t \int_{c-i \infty}^{c+i \infty} \frac{2 t^{\rho+p}(p-1) !(-\rho-p-1) ! \cos [2 \pi r m t]}{\epsilon^{p}(-\rho-1) !} d p
$$

where to ensure convergence of the integration over $t$ the contour must be determined by

$$
\operatorname{Max}(-\rho-1,0)<c=\operatorname{Re}(p)<-\rho .
$$

Interchanging orders of integration and using the identity

$$
\int_{0}^{\infty} t^{\rho+p} \cos [2 \pi r m t] d t=(\rho+p) ! \frac{\cos [\pi(\rho+p+1) / 2]}{(2 \pi r m)^{\rho+p+1}}
$$

$$
-1<\operatorname{Re}(\rho+p)<0,
$$

the expression (2.10) becomes

$$
\begin{aligned}
E_{0}{ }^{[m, 1]} q & =\frac{1}{2 \pi i} \sum_{r=1}^{\infty} \\
& \times \int_{c-i \infty}^{c+i \infty} \frac{2(\rho+p) ! \cos [\pi(\rho+p+1) / 2](-1-\rho-p) !(p-1) !}{(2 \pi r m)^{\rho+p+1}(-\rho-1) ! \epsilon^{p}} d p
\end{aligned}
$$

where the path of integration, $L_{1}$, equivalent to (2.11), can be specified for notational convenience as

$$
L_{1}: \operatorname{Re}(p)=-\rho-\Delta ; \quad 0<\Delta<\operatorname{Min}(-\rho, 1) .
$$

We note that the orders of integration and summation in (2.13) may not be interchanged, as the resulting sum over $r$ would then diverge. However, since the integrand has no poles in the interval

$$
-\rho-\Delta<\operatorname{Re}(p)<1-\rho-\Delta
$$

we may translate the contour to a new path $L_{2}$, given by

$$
L_{2}: \operatorname{Re}(p)=1-\rho-\Delta .
$$

On the new path, since the sum over $r$ is then uniformly convergent, interchange of the order of summation and integration is permissible. The sum over $r$ gives a zeta function, and after using the Riemann relation

$$
2(s-1) ! \zeta(s) \cos (\pi s / 2)=(2 \pi)^{s} \zeta(1-s)
$$

we obtain finally 


$$
E_{0}{ }^{[m, 1]} q=-\frac{1}{2 \pi i} \int_{L_{2}} \frac{\zeta(-\rho-p)(-\rho-p-1) !(p-1) !}{m^{\rho+p+1}(-\rho-1) ! \epsilon^{p}} d p .
$$

If we evaluate the integral by translating the contour to the right, the only poles which contribute are those of $(-\rho-p-1) !$ at $\rho+p=1,3,5, \cdots$. Summing the residues at these poles leads to the descending expansion (2.6). On the other hand an ascending expansion in $(m \epsilon)$ can be found by translating the contour to the left. The poles whose residues contribute to the expansion are those of the zeta function at $p=-\rho-1$ and of the factorial functions at $p=-\rho$, and at $p=0,-1,-2, \cdots$. The result, provided $\rho \neq-1$, is

$$
E_{0}^{[m, 1]} q=\frac{1}{m^{\rho+1}}\left[\frac{(\epsilon m)^{\rho}}{2}-\frac{(\epsilon m)^{\rho+1}}{\rho+1}+\sum_{n=0}^{\infty} \frac{\zeta(n-\rho)}{(\rho-n) !} \rho ! \frac{(\epsilon m)^{n}}{n !}\right] ; \quad \rho \neq-1 .
$$

The expansion converges if $0<\epsilon m<1$. This expansion, and expansion (2.6) are complementary. In most cases, either one or the other are suitable for numerical computation depending on the value of $\epsilon m$. For this simple example, the sum may be expressed in terms of the generalised zeta function (see [1, p. 167]) as

$$
E_{0}^{[m, 1]} q=\frac{1}{m^{\rho+1}}\left[\frac{-(\epsilon m)^{\rho}}{2}-\frac{(\epsilon m)^{\rho+1}}{\rho+1}+\zeta(-\rho, \epsilon m)\right] ; \quad \rho \neq-1 .
$$

The case $\rho=-1$ requires special treatment. Two of the poles of the integrand in (2.18) then coincide at $p=0$. The integrand of (2.18) may be recast into the form

$$
\frac{2 \pi^{2} \cos \pi p / 2}{\sin ^{2} \pi p}\left[\zeta(p) \frac{(2 \pi m \epsilon)^{-p}}{(-p) !}\right] .
$$

The residue of the integrand at the pole at $p=0$ is then

$$
2\left[\frac{d}{d p}\left(\frac{\zeta(p)(2 \pi m \epsilon)^{-p}}{(-p) !}\right)\right]_{p=0}=\ln (m \epsilon)+\gamma
$$

where to obtain this expression we have used the results

$$
\zeta(0)=-\frac{1}{2} ; \zeta^{\prime}(0)=-\frac{1}{2} \ln 2 \pi ;\left[\frac{d}{d p} \ln (p !)\right]_{p=0}=-\gamma=-0.5772 \cdots .
$$

Thus the terms in the expansion (2.19) which correspond to poles of the integrand of (2.18) at $p=-\rho-1$ and $p=0$ are replaced by (2.22) above. This gives

$$
E_{0}^{[m, 1]} q=\frac{1}{2}(\epsilon m)^{-1}+\ln (m \epsilon)+\gamma+\sum_{n=1}^{\infty} \zeta(n+1)(-1)^{n}(\epsilon m)^{n} ; \quad \rho=-1 .
$$

III. $R^{[m, 1]}:\left(x^{\eta}+\epsilon\right)^{\rho}|x|^{\beta}(1-x)^{\omega} h(x)$. We now apply the same technique to obtain the error functional $E^{[m, 1]} q$ for the function

$$
q(x)=\left(x^{\eta}+\epsilon\right)^{\rho}|x|^{\beta}(1-x)^{\omega} h(x), \quad \rho<0, \beta \geqq 0, \omega \geqq 0,
$$

where $h(x)$ and its derivatives are continuous on the interval $[-\epsilon, 1]$. Again in analogy to (2.3) the generalised Poisson summation formula gives

$$
E^{[m, 1]} q=R^{[m, 1]} q-I q=\sum_{r=-\infty}^{\infty} \int_{0}^{1}\left(t^{\eta}+\epsilon\right)^{\rho} t^{\beta}(1-t)^{\omega} h(t) e^{2 \pi i r m t} d t .
$$


However, we may not define $E_{0}^{[m, 1]}$ and $E_{1}{ }^{[m, 1]}$ as in (2.5) as the resulting integrals may diverge. Instead we substitute the integral representation (2.9) for $\left(t^{\eta}+\epsilon\right)^{\rho}$ into (3.2) to obtain

$$
\begin{aligned}
E^{[m, 1]} q= & \frac{1}{2 \pi i} \sum_{r=1}^{\infty} \int_{0}^{1} d t \\
& \times \int_{c=i \infty}^{c+i \infty} \frac{2 t^{\eta(p+\rho)+\beta}(1-t)^{\omega} h(t) \frac{\cos (2 \pi r m t)(-\rho-p-1) !(p-1) !}{(-\rho-1) ! \epsilon^{p}} d p}{(-\infty)}
\end{aligned}
$$

where the orders of integration may be interchanged. An asymptotic expansion as a function of $m$ for the integral over $t$ can be found by the method of Lighthill, and explicitly, using the results of [1, Section 7], we find

$$
\begin{aligned}
E^{[m, 1]} q \cong & \frac{1}{2 \pi i} \sum_{r=1}^{\infty} \int_{c-i \infty}^{c+i \infty} \\
& \times\left\{\sum_{s=0} \frac{\psi_{0}^{(s)}(v)(\eta(p+\rho)+\beta+s) ! 2 \cos [\pi(\eta(p+\rho)+\beta+s+1) / 2]}{s !(2 \pi r m)^{\eta(p+\rho)+\beta+s+1}}\right. \\
& \left.\quad+\sum_{s=0}(-1)^{s} \frac{\psi_{1}^{(s)}(1 ; \eta(p+\rho))(\omega+s) ! 2 \cos [\pi(\omega+s+1) / 2]}{s !(2 \pi r m)^{\omega+s+1}}\right\} \\
& \times \frac{(-\rho-p-1) !(p-1) !}{(-\rho-1) ! \epsilon^{p}} d p
\end{aligned}
$$

where

$$
\begin{aligned}
\psi_{0}(x) & =(1-x)^{\omega} h(x), \\
\psi_{1}(x ; \xi) & =x^{\xi+\beta} h(x) .
\end{aligned}
$$

The path of integration $L_{1}$ may be chosen to be

$$
L_{1}: \operatorname{Re}(p)=-\rho-\Delta
$$

where

$$
\Delta=\frac{1}{2} \operatorname{Min}(-\rho, \beta+1) .
$$

At this stage we separate $E^{[m, 1]} q$ given by (3.4) into two contributions, each corresponding to one of the explicit sums over $s$ in (3.4). Thus

$$
E^{[m, 1]} q=E_{0}^{[m, 1]} q-E_{1}^{[m, 1]} q .
$$

The second of these contributions $E_{1}{ }^{[m, 1]} q$ may be evaluated using the same procedure as that of the previous section. Thus, we first write

$$
\begin{aligned}
-E_{1}{ }^{[m, 1]} q \cong & \frac{1}{2 \pi i} \sum_{r=1}^{\infty} \int_{c-i \infty}^{c+i \infty}\left\{\sum_{s=0}(-1)^{s} \frac{\psi_{1}^{(s)}(1 ; \eta(p+\rho))}{s !}\right. \\
& \left.\times \frac{2(\omega+s) ! \cos [\pi(\omega+s+1) / 2]}{(2 \pi r m)^{\omega+s+1}}\right\} \\
& \times \frac{(-\rho-p-1) !(p-1) !}{(-\rho-1) ! \epsilon^{p}} d p .
\end{aligned}
$$

The orders of summation and integration may be interchanged unless $\omega=0$. In this: 
case the $s=0$ term of the integrand is zero, so that the corresponding term in any expression derived from (3.10) must be omitted. Summing over $r$, and using (2.17), we find

$$
\begin{aligned}
-E_{1}{ }^{[m, 1]} q \cong & \frac{1}{2 \pi i} \int_{c-i \infty}^{c+i \infty} \sum_{s=l} \frac{(-1)^{s} \psi_{1}^{(s)}(1 ; \eta(p+\rho))}{s ! m^{\omega+s+1} \epsilon^{p}} \\
& \times \frac{\zeta(-\omega-s)(-\rho-p-1) !(p-1) !}{(-\rho-1) !} d p
\end{aligned}
$$

where $l=0$ unless $\omega=0$, in which case $l=1$. The poles to the right of the contour are those of $(-\rho-p-1)$ ! situated at $p=-\rho,-\rho+1,-\rho+2, \cdots$. Translating the contour to the right we find the asymptotic expansion

$$
\begin{aligned}
-E_{1}{ }^{[m, 1]} & \cong \sum_{s=l} \sum_{n=0} \frac{(-1)^{s+n} \psi_{1}^{(s)}(1 ; \eta n) \zeta(-\omega-s)(-\rho+n-1) !}{s ! m^{\omega+s+1}(-\rho-1) ! \epsilon^{-\rho+n} n !} \\
& \cong \sum_{s=l} \frac{(-1)^{s} \bar{\psi}_{1}^{(s)}(1) \zeta(-\omega-s)}{s ! m^{\omega+s+1}}
\end{aligned}
$$

where we have written

$$
\bar{\psi}_{1}(x)=\frac{q(x)}{(1-x)^{\omega}}=\left(x^{\eta}+\epsilon\right)^{\rho} x^{\beta} h(x)=\sum_{n=0} \frac{\rho ! \psi_{1}(x ; \eta n)}{n !(\rho-n) ! \epsilon^{-\rho+n}}
$$

and employed the relation

$$
(-1)^{n}(-\rho+n-1) !(\rho-n) !=(-\rho-1) ! \rho !
$$

which is a simple consequence of the standard relation

$$
(z-1) !(-z) ! \sin \pi z=\pi .
$$

Expression (3.12) for $E_{1}{ }^{[m, 1]} q$ is precisely the second part of the expression for $E^{\left[{ }^{m, 1]}\right.} q$ given by the generalised Euler-Maclaurin summation formula (see [1, Eq. (7.8)]), and obtained in [1] by a less cumbersome technique.

The expression for $E_{0}^{[m, 1]} q$ may be handled in the same way. Again for $\beta=0$, the term corresponding to $s=0$ in the summation requires special attention. Provided $\beta>0$, in analogy to (3.11) we find

$$
\begin{aligned}
E_{0}{ }^{[m, 1]} q \cong & \frac{1}{2 \pi i} \int_{c-i \infty}^{c+i \infty} \sum_{s=0} \\
& \times \frac{\psi_{0}^{(s)}(0) \zeta(-\eta(p+\rho)-\beta-s)(-\rho-p-1) !(p-1) !}{s ! m^{\eta(p+\rho)+\beta+s+1} \epsilon^{p}(-\rho-1) !} d p .
\end{aligned}
$$

If we evaluate the integral by completing the contour to the right, we recover the remaining part of the generalised Euler-Maclaurin summation formula, namely

$$
E_{0}{ }^{[m, 1]} q \cong \sum_{s=0} \frac{\bar{\psi}_{0}{ }^{(s)}(0) \zeta(-\beta-s)}{s ! m^{\beta+s+1}}
$$

where

$$
\bar{\psi}_{0}(x)=\left(x^{\eta}+\epsilon\right)^{\rho}(1-x)^{\omega} h(x)=q(x) / x^{\beta} .
$$


Expansion (3.15) suffers from the same defect as expansion (2.6). The terms $\bar{\psi}_{0}^{(s)}(0)$ are large and increase with $s$ because of the proximity of the branch point at $x=-\epsilon$. We therefore proceed as in the previous section, and evaluate (3.15) by translating the contour to the left. The poles of the integrand to the left of the path of integration are those of the factorial function $(p-1)$ !, situated at $p=0,-1$, $-2, \cdots$ and the pole of the zeta function, at $p=-\rho-(\beta+s+1) / \eta$. This pole does not coincide with any of the other poles unless $\rho+(\beta+s+1) / \eta$ is a positive integer or zero. This case requires special treatment which is given in Section 5 . Evaluating the residues at these poles, as in the previous section we have

$$
\begin{aligned}
& E_{\mathrm{C}}{ }^{[m, 1]} q \cong \sum_{s=0} \frac{\psi_{0}{ }^{(s)}(0)}{s ! m^{\eta+\beta+s+1}}\left\{\frac{(((\beta+s+1) / \eta)-1) !((-(\beta+s+1) / \eta)-\rho-1) !}{(-\rho-1) !}\right. \\
& \quad \times\left(\epsilon m^{\eta}\right)^{(\beta+s+1) / \eta+\rho} \\
& \left.+\sum_{n=0} \zeta(\eta(n-\rho)-\beta-s) \frac{\rho !}{(\rho-n) ! n !}\left(\epsilon m^{\eta}\right)^{n}\right\}, \\
& \rho+(\beta+s+1) / \eta \neq \text { integer, } \beta>0 .
\end{aligned}
$$

This expansion is of the same form as the expansion (2.17).

We note that one set of terms here is independent of the value of $m$. In certain cases these terms have a relatively simple interpretation. For example, if $\eta=1$ and if $\rho>-1$, a straightforward calculation indicates that

$$
\begin{aligned}
\frac{\sin [\pi \rho]}{\sin [\pi(\beta+\rho+1)]} \int_{-\epsilon}^{0} q(x) d x & =\frac{\sin [\pi \rho]}{\sin [\pi(\beta+\rho+1)]} \int_{-\epsilon}^{0} \psi_{0}(x)(x+\epsilon)^{\rho}|x|^{\beta} d x \\
& \cong \sum_{s=0} \frac{\psi_{0}^{(s)}(0)}{s !} \frac{(\beta+s) !(-\beta-s-\rho-2) ! \epsilon^{\beta+s+\rho+1}}{(-\rho-1) !} .
\end{aligned}
$$

In such cases, the calculation of the right-hand side may be replaced by a direct calculation of the integral on the left-hand side. This integral has singularities at the end points and as the other given singularity (at $x=1$ ) is relatively distant from the interval of integration [- $-\epsilon, 0]$ the methods of [1] could be applied.

For the special case $\beta=0$, the analysis above requires adjustment. The terms in (3.18) for $s \neq 0$ remain unchanged; for $s=0$, the term is that appearing in Eq. (2.13), of the preceding section. Comparison of the results (2.19) and (3.18) shows that for $\beta=0$ an additional term corresponding to the first term of (2.19) must be included. Thus we find

$$
\begin{aligned}
E_{0}{ }^{[m, 1]}\left(\left(x^{\eta}+\epsilon\right)\right. & \left.(1-x)^{\omega} h(x)\right) \\
& =\lim _{\beta \rightarrow 0+} E_{0}^{[m, 1]}\left(\left(x^{\eta}+\epsilon\right)^{\rho} x^{\beta}(1-x)^{\omega} h(x)\right)-\frac{\psi_{0}(0)\left(\epsilon m^{\eta}\right)^{\rho}}{2 m^{\eta+1}} .
\end{aligned}
$$

The general explanation of this additional term is quite simple. The rule $R^{[m, 1]}$ involves a function evaluation at $x=0$. Thus while in general

$$
\lim _{\beta \rightarrow 0+} f(x) x^{\beta}=f(x), \quad x>0,
$$

in the particular case for which $x=0$

$$
\lim _{\beta \rightarrow 0+} f(x) x=0 .
$$

Thus we have 


$$
\lim _{\beta \rightarrow 0+} R^{[m, 1]}\left(f(x) x^{\beta}\right)=R^{[m, 1]} f(x)-\frac{f(0)}{2 m}
$$

and the term $-f(0) / 2 m$ accounts exactly for the outstanding term in (3.19).

IV. $R_{j} ;\left(x^{\eta}+\epsilon\right)^{\rho} x^{\beta}(1-x)^{\omega} h(x)$. In this section we show how the asymptotic expansion $E_{0}{ }^{[m, 1]} q$ may be generalised for application with a general quadrature rule. The results corresponding to the midpoint rule $R^{[m, 0]}$ may be derived directly from those of the previous section using the identity

$$
R^{[m, 0]}=2 R^{[2 m, 1]}-R^{[m, 1]} .
$$

Although this establishes the results only for the cases in which $\beta$ and $\omega$ are not negative, a separate investigation which is a specialisation of the general results derived below indicates that they remain valid for all $\beta>-1, \omega>-1$.

The most general rule $R$ which we consider is a linear superposition of one point rules $R_{j}$, where

$$
R_{j} f(x)=f\left(t_{j}\right) ; \quad t_{j} \neq 0,1
$$

and $R^{[1,1]}$, where

$$
R^{[1,1]} f(x)=\frac{1}{2}[f(0)+f(1)] .
$$

The expansions for the error functional which correspond to the general rule $R$ are the same linear superposition of the expansions corresponding to these one (and two) point rules. The expansions corresponding to (4.3) are those of Section 3 with $m=1$. We indicate here the construction of expansions for the error functional for the one point rule (4.2).

The generalised Poisson summation formula [1, Eq. (3.4)] states

$$
E q=R_{j} q-I q=\sum_{r=-\infty}^{\infty} d_{r}\left(R_{j}\right) \int_{0}^{1} q(t) \exp [2 \pi i r t] d t
$$

where

$$
d_{r}\left(R_{j}\right)=\exp \left[-2 \pi i r t_{j}\right] .
$$

Thus, substituting the integral representation $(2.9)$ for $(t+\epsilon)^{\rho}$, we find

$$
\begin{aligned}
E q= & \sum_{r=-\infty}^{\infty} d_{r}\left(R_{j}\right) \int_{0}^{1} f(t) \exp [2 \pi i r t] d t \frac{1}{2 \pi i} \\
& \times \int_{c-i \infty}^{c+i \infty} \frac{t^{\eta(\rho+p)}(-\rho-p-1) !(p-1) !}{(-\rho-1) ! \epsilon^{p}} d p
\end{aligned}
$$

where

$$
f(t)=t^{\beta}(1-t)^{\omega} h(t) .
$$

We interchange orders of integration, and for the Fourier transform

$$
g(r)=\int_{0}^{1} t^{\eta(\rho+p)+\beta}(1-t)^{\omega} h(t) \exp [2 \pi i r t] d t
$$

substitute the expansion of [1, Eq. (6.15)], obtaining 


$$
\begin{aligned}
E q \cong & \sum_{r=-\infty}^{\infty} d_{r}\left(R_{j}\right) \frac{1}{2 \pi i} \int_{c-i \infty}^{c+i \infty} \\
& \times\left\{\sum_{s=0} \frac{\psi_{0}{ }^{(s)}(0)(\eta(p+\rho)+\beta+s) !}{s !(2 \pi r)^{\eta(p+\rho)+\beta+s+1}} \exp [\pi i(\eta(\rho+p)+\beta+s+1) / 2]\right. \\
& \left.\quad+\sum_{s=0}(-1)^{s} \frac{\psi_{1}^{(s)}(1 ; \eta(p+\rho))(\omega+s) !}{s !(2 \pi r)^{\omega+s+1}} \exp [-\pi i(\omega+s+1) / 2]\right\} \\
& \times \frac{(-\rho-p-1) !(p-1) !}{(-\rho-1) ! \epsilon^{p}} d p
\end{aligned}
$$

where $\psi_{0}(x)$ and $\psi_{1}(x ; \xi)$ are given by (3.5) and (3.6), and the path of integration is $L_{1}$ given by (3.7) and (3.8).

With the exception of the first terms $(s=0)$, we may interchange the orders of summation and integration, and carry out the sum over $r$ which is absolutely convergent. For the first terms $(s=0)$, the summation over $r$ is only conditionally convergent. However, a more detailed investigation indicates that for this case too the interchange is permissible. This may be shown by translating the contour to the right, and including a contribution from the residue of the pole at $p=-\rho$. After carrying out the sum, we may then move the contour back to its original position, and include again a contribution from the residue at the pole at $p=-\rho$. These two contributions cancel. This procedure depends critically on $d_{r}\left(R_{j}\right)$ being given by (4.5) with $t_{j} \neq 0,1$. We may therefore change orders of summation and integration in (4.9). This is precisely equivalent to interchanging orders of summation and integration in (4.6) from which (4.9) was derived. We may therefore write

$$
\begin{aligned}
E q= & \frac{1}{2 \pi i} \int_{c-i \infty}^{c+i \infty} d p \sum_{r=-\infty}^{\infty} d_{r}\left(R_{j}\right) \int_{0}^{1} t^{\eta(\rho+p)} f(t) \exp [2 \pi i r t] d t \\
& \times \frac{(-\rho-p-1) !(p-1) !}{(-\rho-1) ! \epsilon^{p}}
\end{aligned}
$$

That part of the integrand which involves the sum over $r$ and the integral over $t$ has been investigated in detail in [1]. Referring to the generalised Poisson summation formula, it is simply an error functional which corresponds to the function

$$
\begin{aligned}
\bar{f}(x ; \eta(\rho+p)) & =f(x) x^{\eta(\rho+p)}=q(x)\left(x^{\eta}+\epsilon\right)^{-\rho} x^{\eta(\rho+p)} \\
& =x^{\beta+\eta(\rho+p)}(1-x)^{\omega} h(x) .
\end{aligned}
$$

Thus we have the relation

$$
E q=R_{j} q-I q=\frac{1}{2 \pi i} \int_{c-i \infty}^{c+i \infty}\left(R_{j} \bar{f}-I \bar{f}\right) \frac{(-\rho-p-1) !(p-1) !}{(-\rho-1) ! \epsilon^{p}} d p
$$

where $q$ and $\bar{f}$ are related by (4.11). The relation (4.12) is true also if $R_{j}$ is the end point rule (4.3) and $\beta>0$, but does not hold if $R$, is the end point rule and $\beta=0$.

An asymptotic expansion for $R_{j} \bar{f}-I \bar{f}$ is given in [1] by the generalised EulerMaclaurin expansion equations (8.1) and (8.2) which we substitute into (4.12). We may then write

$$
E q=R_{j} q-I q=E_{0} q-E_{1} q
$$

where 


$$
\begin{aligned}
E_{0} \cong \cong & \frac{1}{2 \pi i} \int_{c-i \infty}^{c+i \infty} \sum_{s=0} \\
& \times \frac{\psi_{0}^{(s)}(0) \zeta\left(-\eta(p+\rho)-\beta-s ; t_{j}\right)(-\rho-p-1) !(p-1) !}{s !(-\rho-1) ! \epsilon^{p}} d p .
\end{aligned}
$$

If again we close the contour of (4.12) to the right, we recover the generalised EulerMaclaurin formula [1, Eq. (8.1)]. If we close the contour in (4.14) to the left, we recover the appropriate generalisation of (3.18). The result is

$$
\begin{aligned}
E_{0} q \cong \sum_{s=0} \frac{\psi_{0}{ }^{(s)}(0)}{s !}\left\{\frac{(((\beta+s+1) / \eta)-1) !(-((\beta+s+1) / \eta)-\rho-1) !}{(-\rho-1) !}\right. & \\
(4.15) & \left.\times \epsilon^{((\beta+s+1) / \eta)+\rho}+\sum_{n=0} \zeta\left(\eta(n-\rho)-\beta-s, t_{j}\right) \frac{\rho !}{(\rho-n) ! n !} \epsilon^{n}\right\}, \\
\rho+(\beta+s+1) / \eta \neq \text { integer, } &
\end{aligned}
$$

where the restriction on $\rho+(\beta+s+1) / \eta$ arises in the same way as in the corresponding Eq. (3.18).

V. Special Values of the Parameters $\beta$ and $\rho$. The terms in the expansions for $E_{0} q$ in which $\rho+(\beta+s+1) / \eta$ is a positive integer or zero require further attention. The coefficient of $\epsilon^{\rho+(\beta+s+1) / \eta}$ in (4.15) or (3.18) is in this case the sum of two terms, each of which is indeterminate. We may calculate the residue of the integrand in (4.14) or (3.15) at $p=-\rho-(\beta+s+1) / \eta$ which is due to a double pole, the coincidence of a simple pole of $(p-1)$ ! and a simple pole of the generalised zeta function. We make use of the results

$$
\begin{aligned}
\lim _{\delta \rightarrow 0}\left(\zeta\left(1+\delta, t_{j}\right)-\frac{1}{\delta}\right) & =-\Psi\left(t_{j}-1\right), \\
\lim _{\delta \rightarrow 0}\left((-N-1+\delta) !-\frac{(-1)^{N}}{\delta N !}\right) & =(-1)^{N} \frac{\Psi(N)}{N !}, \\
\lim _{\delta \rightarrow 0} \frac{\left(\epsilon m^{\eta}\right)^{-\delta}-1}{\delta} & =-\ln \left(\epsilon m^{\eta}\right),
\end{aligned}
$$

where

$$
\Psi(z)=d(\ln z !) / d z .
$$

This leads to a residue of the form $\psi_{0}^{(s)}(0) R_{s} / s ! m^{\eta \rho+\beta+s+1}$ where

$$
\begin{aligned}
& R_{s}=-\frac{(-1)^{N}(-\rho+N-1) !(\epsilon m)^{N}}{\eta N !(-\rho-1) !}\{\psi(N)-\psi(-\rho+N-1) \\
& N=\rho+(\beta+s+1) / \eta=\text { integer } \geqq 0 .
\end{aligned}
$$

To modify (3.18) we set $t_{j}=1$ and to modify (4.15) we set $m=1 \mathrm{in}$ this and the subsequent expressions. Either equation may be written

$$
\text { (5.6) } E_{0} q \cong \sum_{s=0} \frac{\psi_{0}^{(s)}(0)}{s ! m^{\eta \rho+\beta+s+1}}\left[R_{s}+\sum_{n=0}^{\prime} \zeta\left(\eta(n-\rho)-\beta-s, t_{j}\right) \frac{\rho !}{(\rho-n) ! n !}\left(\epsilon m^{\eta}\right)^{n}\right]
$$

where the prime on the summation symbol indicates that if $\rho+(\beta+s+1) / \eta=N$, 
an integer, the term $n=N$ is to be omitted from the summation. In this case $R_{8}$ is given by (5.5) above. Otherwise it takes the value corresponding to the first term of (3.18) or (4.15), namely

$$
\text { (5.7) } \begin{array}{r}
R_{s}=(((\beta+s+1) / \eta)-1) !((-(\beta+s+1) / \eta)-\rho-1) ! \frac{\left(\epsilon m^{\eta}\right)^{\rho+(\beta+s+1) / \eta}}{(-\rho-1) !}, \\
\rho+(\beta+s+1) / \eta \neq \text { integer }
\end{array}
$$

VI. $R:(x+\epsilon \exp [i \theta])^{\rho / 2}(x+\epsilon \exp [-i \theta])^{\rho / 2} x^{\beta}(1-x)^{\omega} h(x)$. In applications in theoretical physics, integrands of the type $f(t) /\left(t^{2}+\epsilon^{2}\right)^{\nu}$ or $f(t) /\left[(t-\alpha)^{2}+\epsilon^{2}\right]^{\nu}$ or products of such functions frequently occur. This is particularly so in applications which involve field theory, dispersion relations and the many body problem. Factors of the form $\left[(t-\alpha)^{2}+\epsilon^{2}\right]^{\nu}$ arise in perturbation theories as 'energy denominators' or propagators. Usually these integrals cannot be evaluated analytically. We investigate in this section the asymptotic expansions associated with the error functional of integrals of this form.

We consider the function

$$
q(t)=f(t)(t+\epsilon \exp [i \theta])^{\rho / 2}(t+\epsilon \exp [-i \theta])^{\rho / 2}
$$

where as before, $f(t)$ is of the form

$$
f(t)=t^{\beta}(1-t)^{\omega} h(t)
$$

and $h(t)$ and its derivatives are continuous throughout the interval of integration. We use the result (Erdélyi et al. [3])

$$
\begin{aligned}
&\left(1+2 x \cos \theta+x^{2}\right)^{\rho / 2}= \frac{1}{2 \pi i} \int_{c-i \infty}^{c+i \infty} x^{p+\rho}(2 \sin \theta)^{-(\rho+1) / 2}(-(\rho+1) / 2) ! \\
& \times \frac{(p-1) !(-\rho-p-1) !}{(-\rho-1) !} P_{p+(\rho-1) / 2}^{(\rho+1) / 2}(\cos \theta) d p, \\
& 0<\operatorname{Re}(p)<-\rho
\end{aligned}
$$

where $P_{\mu}{ }^{\lambda}$ is the associated Legendre function of the first kind. Applying the generalised Poisson summation formula, we find without difficulty that

$$
\begin{aligned}
R q-I q & =\sum_{r=-\infty}^{\infty} d_{r}(R) \int_{0}^{1} f(t) e^{2 \pi i r t} d t \frac{1}{2 \pi i} \int_{c-i \infty}^{c+i \infty} t^{p+\rho}(2 \sin \theta)^{-(\rho+1) / 2} \\
& \times \frac{(-(\rho+1) / 2) !(p-1) !(-\rho-p-1) !}{(-\rho-1) ! \epsilon^{p}} P_{p+(\rho-1) / 2}^{(\rho+1) / 2}(\cos \theta) d p .
\end{aligned}
$$

This expression differs only in detail from expression (4.6) if we set $\eta=1$. The integration over the $t$ variable is identical, and the integration over the $p$ variable has to be adjusted to take into account the associated Legendre polynomial. Since this has no poles in the $p$ plane, the net result is that each term in the ensuing expansions need only be adjusted by the multiplicative factor $P_{p+(\rho-1) / 2}^{(p+1) / 2}(\cos \theta)$. Finally the whole expansion has to include an overall factor

$$
(2 \sin \theta)^{-(\rho+1) / 2}(-(\rho+1) / 2) ! .
$$

The results corresponding to Eqs. (2.19), (3.18), and (4.15) are as follows. If $f(t)=1$ 


$$
\begin{aligned}
E_{0}{ }^{[m, 1]} q= & \frac{(2 \sin \theta)^{-(\rho+1) / 2}}{m^{\rho+1}}(-(\rho+1) / 2) ! \\
\times & {\left[P_{-(\rho+1) / 2}^{(\rho+1) / 2}(\cos \theta) \frac{(\epsilon m)^{\rho}}{2}-P_{-(\rho+3) / 2}^{(\rho+1) / 2}(\cos \theta) \frac{(\epsilon m)^{\rho+1}}{(\rho+1)}\right.} \\
& \left.\quad+\sum_{n=0} \frac{\zeta(n-\rho)}{(\rho-n) ! n !} \rho ! P_{(\rho-1-2 n) / 2}^{(\rho+1) / 2}(\cos \theta)(\epsilon m)^{n}\right] ; \quad f(t)=1 .
\end{aligned}
$$

For $f(t)$ given by (6.2) above with $\beta>0$

$$
\begin{aligned}
E_{0}{ }^{[m, 1]} q \cong & \sum_{s=0} \frac{\psi_{0}{ }^{(s)}(0)}{s !} \frac{(2 \sin \theta)^{-(\rho+1) / 2}(-(\rho+1) / 2) !}{m^{\rho+\beta+s+1}} \\
& \times\left[\frac{(\beta+s) !(-\beta-s-2-\rho) !}{(-\rho-1) !} P_{-\beta-s-(\rho+3) / 2}^{(\rho+1) / 2}(\cos \theta)(\epsilon m)^{\rho+\beta+s+1}\right. \\
& \left.+\sum_{n=0} \frac{\zeta(-\rho-\beta-s+n)}{(\rho-n) ! n !} \rho ! P_{(\rho-1-2 n) / 2}^{(\rho+1) / 2}(\cos \theta)(\epsilon m)^{n}\right], \\
& (\rho+\beta) \neq \text { integer. }
\end{aligned}
$$

The corresponding expansion for the one point rule $R_{j} f=f\left(t_{j}\right)$ is the same as (6.6) except that $\zeta(-\rho-\beta-s+n)$ is replaced by $\zeta\left(-\rho-\beta-s+n, t_{j}\right)$ and $m$ is replaced by 1 .

If $\rho+\beta$ is an integer, we may proceed as in Section 5 . We require in addition to the quantities listed there, the derivative of the Legendre function with respect to the lower argument, namely

$$
\frac{d}{d p}\left[P_{p+(\rho-1) / 2}^{(\rho+1) / 2}(\cos \theta)\right]_{p=-\rho-\beta-s-1} .
$$

In this case expansion (6.6) is replaced by

$$
\begin{aligned}
E_{0}{ }^{[m, 1]} q \cong & \sum_{s=0} \frac{\psi_{0}{ }^{(s)}(0)(2 \sin \theta)^{-(\rho+1) / 2}}{s ! m^{\rho+\beta+8+1}}(-(\rho+1) / 2) ! \\
& \times\left[R_{s}+\sum_{n=0}^{\prime} \zeta(-\rho+n-\beta-s) \frac{\rho !}{(\rho-n) ! n !} P_{(\rho-1-2 n) / 2}^{(\rho+1) / 2}(\cos \theta)(\epsilon m)^{n}\right]
\end{aligned}
$$

where

$$
\begin{aligned}
& R_{s}=-\frac{(-1)^{N}(-\rho+N-1) !(\epsilon m)^{N}}{N !(-\rho-1) !} P_{-N+(\rho-1) / 2}^{(\rho+1) / 2}(\cos \theta) \\
& (6.8) \times\left\{\psi(N)-\psi(N-\rho-1)+\psi(0)-\ln (\epsilon m)+\left[\frac{d}{d p} \ln P_{p+(\rho-1) / 2}^{(\rho+1) / 2}(\cos \theta)\right]_{p=-N}\right\}, \\
& N=\rho+\beta+s+1=\text { integer } \geqq 0 .
\end{aligned}
$$

As in Section 5, the prime on the summation symbol indicates that if $\rho+\beta+s+1$ is a nonnegative integer $N$, the term $n=N$ is to be omitted from the sum and $R_{s}$ is then given by (6.8). 
Applied Mathematics Department University of New South Wales

Kensington, Australia

Applied Mathematics Division Argonne National Laboratory Argonne, Illinois 60439

1. J. N. Lyness \& B. W. NinhaM, "Asymptotic expansions and numerical quadrature," Math. Comp., v. 21, 1967, pp. 162-178.

2. A. ERdélyi, W. Magnus, F. Oberhettinger \& F. G. Tricomi, Higher Transcendental Functions, Vol. 1, McGraw-Hill, New York, 1953. MR 15, 419.

3. A. Erdélyi, W. Magnus, F. Oberhettinger \& F. G. Tricomi, Tables of Integral Transforms, Vol. 1, McGraw-Hill, New York, 1954, p. 310. MR 15, 868. 